\title{
Statistical Survey of Type III Radio Bursts at Long Wavelengths Observed by the Solar TErrestrial RElations Observatory (STEREO)/Waves Instruments: Goniopolarimetric Properties and Radio Source Locations
}

\author{
V. $\operatorname{Krupar}^{1} \cdot$ M. Maksimovic ${ }^{2}$. \\ O. Santolik ${ }^{1,3}$. B. Cecconi ${ }^{2}$. O. Kruparova ${ }^{1}$ \\ (C) Springer $\bullet \bullet \bullet \bullet$
}

\begin{abstract}
We have performed statistical analysis of a large number of Type III radio bursts observed by STEREO between May 2007 and February 2013. Only intense, simple, and isolated cases have been included in our data set. We have focused on the goniopolarimetric (GP, also referred to as direction-finding) properties at frequencies between $125 \mathrm{kHz}$ and $2 \mathrm{MHz}$. The apparent source size $\gamma$ is very extended $\left(\approx 60^{\circ}\right)$ for the lowest analyzed frequencies. Observed apparent source sizes $\gamma$ expand linearly with a radial distance from the Sun at frequencies below $1 \mathrm{MHz}$. We have shown that Type III radio bursts statistically propagate in the ecliptic plane. Calculated positions of radio sources suggest that scattering of the primary beam pattern plays an important role in the propagation of Type III radio bursts in the IP medium.
\end{abstract}

Keywords: Plasma radiation, Solar radio emissions

\section{Introduction}

Solar radio emissions represent a clear evidence of far-reaching effects of solar eruptions injecting energetic electrons into the interplanetary (IP) medium. Kinetic energy of these electrons can be partly converted into electromagnetic radiation. Therefore solar radio emissions provide us with an important diagnostic tool for remote measurements of the electron density and magnetic field between the Sun and the Earth. These emissions are often called radio bursts to emphasize their brief and explosive characteristics.

\footnotetext{
$\overline{1}$ Institute of Atmospheric Physics ASCR, Bocni II 1403, Prague 141 31, Czech Republic email: vk@ufa.cas.cz

2 LESIA, UMR CNRS 8109, Observatoire de Paris, Meudon 92195, France

3 Faculty of Mathematics and Physics, Charles University,

Ke Karlovu 3, Prague 121 16, Czech Republic
} 
Type III radio bursts belong among the most intense electromagnetic emissions observed in the heliosphere (Wild, 1950, Dulk et al. 1987). They are consequences of impulsively accelerated electrons associated with solar flares. The mechanism of energy release in solar flares is believed to be related to the reconnection of the magnetic field lines. The magnetic field creates the current sheet representing a very efficient particle accelerator (Martens and Kuin, 1989). The downward moving electrons radiate in the X-ray spectra, whereas the electrons propagating outward from the Sun along open magnetic field lines can produce an intense radio emission: Type III radio bursts.

Type III-generating electron beams produce a bump-on-tail instability of the electron velocity distribution function (Ginzburg and Zhelezniakov, 1958; Melrose, 1980). This abrupt change in plasma parameters trigger electrostatic Langmuir waves at the local plasma frequency $f_{\text {pe }}$, which is proportional to the square root of the local electron density $\left(f_{\mathrm{pe}}[\mathrm{kHz}] \approx 9 \sqrt{n_{\mathrm{e}}\left[\mathrm{cm}^{-3}\right]}\right)$. Langmuir waves can be afterward converted by nonlinear interactions into Type III radio bursts (Gurnett and Anderson, 1976, Lin et al., 1981). Langmuir waves scattered off of ions or low-frequency turbulence result in electromagnetic waves at $f_{\text {pe }}$ (the fundamental emission). Two Langmuir waves can coalesce to produce radiation at $2 f_{\text {pe }}$ (the harmonic emission). This generation mechanism is often referred to as the plasma emission mechanism. Since electrons propagate outward from the Sun to lower densities, these emissions are generated at lower frequencies corresponding to a decreasing in $f_{\mathrm{pe}}$. Therefore the locations of Type III radio bursts enable us to track solar energetic particles and to map the global magnetic field topology in the corona and IP medium. Measurements of IP Type III radio bursts from ground-based observatories are restricted by the ionospheric cut-off frequency and radio instruments in space are thus needed despite their high requirements on design.

Radio sources can be tracked with one spacecraft with goniopolarimetric (GP, also referred to as direction-finding) capabilities using an average IP density model (Fainberg, Evans, and Stone, 1972). However, the IP density profile along the path of exciter electrons is not obvious at the given time and location. In a case of long-lasting Type III storm we may localize radio sources using solar rotation (Bougeret, Fainberg, and Stone, 1984). Multi-spacecraft observations allow to triangulate radio sources without any additional assumptions on the IP density model (Weber et al., 1977; Fitzenreiter et al., 1977; Reiner et al., 1998, 2009). The Solar TErrestrial RElations Observatory (STEREO) is the first three-axis stabilized solar mission that consists of two spacecraft embarking identical radio receivers which enable us to accurately track source locations of solar radio emissions (Kaiser et al., 2008, Cecconi et al., 2008).

This is the second of two linked papers that summarize our findings on statistical properties of Type III radio bursts observed by the STEREO/Waves instruments. In this paper we focus on GP properties. The frequency spectra and its relation to electron beams producing these bursts have been discussed in the first paper (Krupar et al. 2014 ). In Section 2 we describe source data set and methods used. In Section 3 we present a detailed analysis of two Type III radio bursts as an example of our data set. We discuss statistical results of a large number of Type III radio bursts. In Section 4 we summarize our findings and we make concluding remarks. 


\section{Instrumentation and Methodology}

\subsection{The STEREO/Waves Instrument}

The STEREO/Waves instrument provides comprehensive measurements of the three components of the electric field fluctuations up to $16 \mathrm{MHz}$ (Bougeret et al. 2008). Three mutually orthogonal monopole antenna elements (each six meters in length) form the sensor part of the STEREO/Waves instrument (Bale et al., 2008). The High Frequency Receiver (HFR, a part of STEREO/Waves) has instantaneous GP capabilities between $125 \mathrm{kHz}$ and $2 \mathrm{MHz}$ encompassing both the direction-finding and polarization (Cecconi et al., 2008).

The STEREO/Waves effective antenna directions and lengths are different from the physical ones due to their electric coupling with the spacecraft body. The galactic background, as a nearly stable isotropic source, allowed us to determine reduced effective antenna lengths (Zaslavsky et al. , 2011). The effective antenna directions have been obtained by observations of the auroral kilometric radiation (AKR) during STEREO-B roll maneuvers (Krupar et al. 2012 ). As no AKR has been surveyed by STEREO-A, the effective directions have been assumed to be the same.

\subsection{Singular Value Decomposition of the Electric Spectral Matrix}

The singular value decomposition (SVD) is an iterative method for a wave analysis of multi-component measurements of the magnetic field with a point source (Santolík, Parrot, and Lefeuvre, 2003). We may extend its application to electric field measurements when a wave frequency $f$ is significantly larger than the local electron plasma frequency $f_{\mathrm{pe}}$, which is the case of radio waves observed by STEREO/Waves/HFR (Krupar et al., 2010, 2012).

Applying SVD on the spectral matrix A composed from electric complex amplitudes $\hat{E}_{k}$ with separated real and imaginary parts we obtain matrices $\mathbf{U}$, $\mathbf{W}$, and $\mathbf{V}^{\mathrm{T}}$ :

$$
\mathbf{A}=\mathbf{U} \cdot \mathbf{W} \cdot \mathbf{V}^{\mathrm{T}} .
$$

The diagonal nonnegative matrix $\mathbf{W}$ in Equation (1) contains three singular values that represent relative lengths of the axes of the polarization ellipsoid in ascending order: $w_{1}, w_{2}$, and $w_{3}$ are minimal, medial, and maximal singular values. These singular values reveal electric field variances along axes of the polarization ellipsoid. In a case of a monochromatic plane wave with a point source the polarization ellipsoid degenerates to an ellipse $\left(w_{1} / w_{3}=0\right)$. On the other hand if no direction is preferred $\left(w_{1}=w_{2}=w_{3}\right)$, the polarization ellipsoid degenerates and becomes a sphere. Therefore we assume that information about the angular half aperture of the source $(\gamma)$ as seen by the spacecraft is hidden in the ratio $w_{1} / w_{3}$. We have used an empirical relation between $w_{1} / w_{3}$ and $\gamma$ considering unpolarized emissions and the Gaussian radial source shape (Krupar et al. 2012).

SVD provides us with three characteristic directions of the polarization ellipsoid in the rows of the $\mathbf{V}^{\mathrm{T}}$ matrix: the wave vector, minor polarization axis, and 
major polarization axis directions. These three directions are mutually orthogonal. The wave vector direction $\boldsymbol{\kappa}=\mathbf{k} /|\mathbf{k}|$ is in the row that corresponds to $w_{1}$, i.e. to the shortest axis of the polarization ellipsoid.

The degree of polarization describes a quantity of an electromagnetic wave which is polarized. We use the two-dimensional (2D) degree of polarization in the polarization plane $(C)$ according to Santolík et al. (2002):

$$
C=\sqrt{2 \frac{R_{33}^{2}+R_{22}^{2}+2\left|R_{23}\right|}{R_{33}+R_{22}}-1},
$$

where $R_{i j}$ is the spectral matrix $A_{i j}$ transformed into a matrix in the primary axial system (i.e. the polarization plane):

$$
\begin{array}{r}
R_{k l}=\sum_{m=1}^{3} W_{l l} U_{m l} V_{m k} \text { for } k=1,2,3 \text { and } l=1,2,3, \\
R_{k l}=\sum_{m=4}^{6} W_{l l} U_{m l} V_{m-3 k-3} \text { for } k=4,5,6 \text { and } l=1,2,3 .
\end{array}
$$

In this paper for a localization of radio sources we use the heliocentric earth equatorial (HEEQ) coordinate system with the following definition: the $Z_{\mathrm{HEEQ}}$ axis denotes the solar rotation axis, and the $X_{\mathrm{HEEQ}}$ axis is in the plane containing the $Z_{\mathrm{HEEQ}}$ axis and Earth. For relative wave directions we use the radialtangential-normal $(\mathrm{RTN})$ coordinate system. The $X_{\mathrm{RTN}}$ axis points from the spacecraft to the Sun's center, and the $Y_{\mathrm{RTN}}$ axis is the cross product of the solar rotational axis and $X_{\mathrm{RTN}}$ lying in the solar equatorial plane towards the West limb.

\subsection{Triangulation}

Aforementioned determination of the wave vector direction $\kappa$ allows us to estimate a source location if an electromagnetic emission is observed by two (or more) spacecraft. This process is referred to as the triangulation. The source location can be found as an intersection of lines $\mathbf{L}_{\mathrm{A}}$ and $\mathbf{L}_{\mathrm{B}}$ in three dimensional (3D) space (Figure 1):

$$
\begin{aligned}
& \mathbf{L}_{\mathrm{A}}=\mathbf{P}_{\mathrm{A}}+t_{\mathrm{A}} \boldsymbol{\kappa}_{\mathrm{A}}, \\
& \mathbf{L}_{\mathrm{B}}=\mathbf{P}_{\mathrm{B}}+t_{\mathrm{B}} \boldsymbol{\kappa}_{\mathrm{B}},
\end{aligned}
$$

where $\mathbf{P}_{k}$ denotes spacecraft position vector, $\boldsymbol{\kappa}_{k}$ represents a wave vector direction and $t_{k}$ is a parameter we need to retrieve. Two lines intersect in 3D at one point only if they lie in the same plane. In a general case, we have to determine the closest point on line $\mathrm{A}\left(\mathbf{r}_{\mathrm{A}}\right)$ and the closest point on line $\mathrm{B}\left(\mathbf{r}_{\mathrm{B}}\right)$. First we calculate the direction between two spacecraft $\left(\mathbf{P}_{\mathrm{BA}}\right)$ and the direction 
perpendicular to both wave vector directions $(\mathbf{M})$ :

$$
\begin{array}{r}
\mathbf{P}_{\mathrm{BA}}=\mathbf{P}_{\mathrm{B}}-\mathbf{P}_{\mathrm{A}}, \\
\mathbf{M}=\boldsymbol{\kappa}_{\mathrm{B}} \times \boldsymbol{\kappa}_{\mathrm{A}} .
\end{array}
$$

Then we calculate the direction $\mathbf{D}$ which is perpendicular to $\mathbf{P}_{\mathrm{BA}}$ and $\mathbf{M}$ :

$$
\mathbf{D}=\frac{\mathbf{P}_{\mathrm{BA}} \times \mathbf{M}}{\|\mathbf{M}\|^{2}}
$$

From $\mathbf{D}$ we can derive parameters $t_{\mathrm{AI}}$ and $t_{\mathrm{BI}}$ as its projections into the mutual wave vector directions:

$$
\begin{aligned}
& t_{\mathrm{AI}}=\mathbf{D} \cdot \boldsymbol{\kappa}_{\mathrm{B}}, \\
& t_{\mathrm{BI}}=\mathbf{D} \cdot \boldsymbol{\kappa}_{\mathrm{A}} .
\end{aligned}
$$

Finally, we obtain the closest points on each of lines:

$$
\begin{aligned}
\mathbf{r}_{\mathrm{A}} & =\mathbf{P}_{\mathrm{A}}+t_{\mathrm{AI}} \boldsymbol{\kappa}_{\mathrm{A}}, \\
\mathbf{r}_{\mathrm{B}} & =\mathbf{P}_{\mathrm{B}}+t_{\mathrm{BI}} \boldsymbol{\kappa}_{\mathrm{B}} .
\end{aligned}
$$

In this paper we consider the intersection $(\mathbf{r})$ to be the closest point between two lines which can be calculated as a mean distance between $\mathbf{r}_{\mathrm{A}}$ and $\mathbf{r}_{\mathrm{B}}$ :

$$
\mathbf{r}=\frac{\mathbf{r}_{\mathrm{A}}+\mathbf{r}_{\mathrm{B}}}{2}
$$

Projections of a segment line $\mathbf{r}_{\mathrm{A}}$ and $\mathbf{r}_{\mathrm{B}}$ reveal an accuracy of the triangulation $\left(e_{\mathrm{i}}\right)$ in the given direction:

$$
e_{\mathrm{i}}=\left|r_{\mathrm{Ai}}-r_{\mathrm{Bi}}\right|
$$

\section{Results and Discussion}

We have manually selected 152 time-frequency intervals when Type III radio bursts have been observed by STEREO/Waves between May 2007 and February 2013. The separation angle between the spacecraft in the ecliptic plane ranged between $7^{\circ}$ (May 2007) and $180^{\circ}$ (February 2011). We have included only simple and isolated events when flux density $S$ was intense enough for the GP analysis. We have linked $20 \%$ of Type III radio bursts with solar flares using database compiled by the Lockheed Martin Solar and Astrophysics Laboratory (http:// www.Imsal.com/solarsoft/latest_events_archive.html). A detailed description of the data set can be found in the first article (Krupar et al., 2014). As an example from our list of events we present two Type III radio bursts observed in January 2008 and in May 2009. 


\subsection{January 2008 Type III Burst}

We show an observation of a Type III radio burst from 29 January 2008, which has been already analyzed by Reiner et al. (2009) and Martínez-Oliveros et al. (2012). It has been linked to a B1.2 solar flare starting at 17:28 UT reaching maximum at 17:43 UT. The flare site was at $\mathrm{S} 09^{\circ} \mathrm{E} 59^{\circ}$ in the HEEQ coordinate system. During this event STEREO-A was located $21.6^{\circ}$ west from a Sun-Earth line at $0.97 \mathrm{AU}$ from the Sun whereas STEREO-B was at $23.6^{\circ}$ east and $1.00 \mathrm{AU}$ from the Sun. Figures 2 and 3 show the data recorded on STEREO-A and STEREO-B, respectively. Both STEREO detected a simple and isolated Type III radio burst with a starting time of about 17:28 UT. The signal measured by STEREO-B was significantly larger than by STEREO-A which suggests that radio source is located closer to STEREO-B.

We have identified data points corresponding to peak fluxes for each frequency separately (Figure 4). The flux density $S_{\mathrm{B}}$ is about of one order of magnitude larger than $S_{\mathrm{A}}$ with the maximum at $475 \mathrm{kHz}$ at both spacecraft. The flux density $S_{\mathrm{A}}$ for the lowest frequency channel is very weak $\left(\approx 5 \times 10^{-19} \mathrm{Wm}^{-2} \mathrm{~Hz}^{-1}\right)$ being comparable with background. The apparent source sizes $\gamma_{\mathrm{A}}$ and $\gamma_{\mathrm{B}}$ are very extended at both spacecraft. While $\gamma_{\mathrm{B}}$ is largest for the lowest frequency channels corresponding to previous studies (Steinberg, Hoang, and Dulk, 1985), $\gamma_{\mathrm{A}}$ exhibits no frequency dependence. It can be explained by weak $S_{\mathrm{A}}$ when the planarity of the polarization, used for $\gamma$ estimation, is dominated by background signals. The wave vector directions $\theta_{\mathrm{A}} / \theta_{\mathrm{B}}$ (the Sun direction: $\theta=90^{\circ}$, the southward direction: $\theta=0^{\circ}$, and the northward direction: $\left.\theta=180^{\circ}\right)$ and $\phi_{\mathrm{A}} / \phi_{\mathrm{B}}$ (the Sun direction: $\phi=0^{\circ}$, the eastward direction: $\phi=90^{\circ}$, and the westward direction: $\phi=-90^{\circ}$ ) point roughly towards the Sun. Only the low frequency part of $\phi_{\mathrm{A}} / \phi_{\mathrm{B}}$ shows an eastward shift which corresponds to the flare site. The highest frequency channel $(1975 \mathrm{kHz})$ is significantly affected by instrumental effects when HFR switches from the three monopoles mode (HFR1, GP data) to the dipole/monopole mode (HFR2, no GP data). The last panel of Figure 4 contains the $2 \mathrm{D}$ degree of polarization which is about 0.2 at STEREO-A and 0.1 at STEREO-B that is comparable with limits of an accuracy of the receiver itself. The increase at $125 \mathrm{kHz}$ at STEREO-A is probably related to the weak signal.

We have performed a triangulation of radio sources (Section 2.3. using wave vector directions during peak fluxes (Figure 5). The accuracy of radio source locations calculated from Equation (15) is significantly larger in the $X Y_{\text {HEEQ }}$ plane than in the $X Z_{\mathrm{HEEQ}}$ plane due to the smaller separation angle between the two STEREO in the latter. We have also included the Parker spiral rooted in the flare site $\left(\mathrm{S} 09^{\circ} \mathrm{E} 59^{\circ}\right)$ assuming a typical solar wind speed of $500 \mathrm{~km} \mathrm{~s}^{-1}$ to illustrate the path followed by the electron beam (Parker, 1958). We have achieved a good agreement between the Parker spiral and radio locations for frequencies above $700 \mathrm{kHz}$. We suggest that the low frequency part is shifted due to propagation effects in the IP medium (Steinberg et al., 1984, Thejappa, MacDowall, and Kaiser, 2007). The dotted ellipses around three intersections $(175 \mathrm{kHz}, 1075 \mathrm{kHz}$, and $1925 \mathrm{kHz}$ ) represent the apparent source sizes as seen from both spacecraft. The two dashed-dotted segment lines show linear source sizes were obtained from 
a source location, $\gamma_{A}$, and $\gamma_{B}$ for $175 \mathrm{kHz}$. The ellipse around these segment lines is the smaller one from the only two possible solutions. We have included the $1975 \mathrm{kHz}$ frequency channel (located at $[0.8,0.0,-0.1]_{\mathrm{HEEQ}}$ ) to illustrate the aforementioned instrumental effects of HFR switching. The error bar on the $125 \mathrm{kHz}$ frequency channel (located at $[0.8,-0.2,0.1]_{\mathrm{HEEQ}}$ ) indicates that the obtained radio source location is unreliable.

Results of the triangulation confirm an assumption that suprathermal electrons triggering the Type III burst propagate in the ecliptic (Dulk et al., 1986). Source regions of higher frequencies are located closer to the Sun as it can be expected. We conclude that calculated radio sources are located closer to STEREO-B being in agreement with location of the solar flare site $\left(\mathrm{S} 09^{\circ} \mathrm{E}^{\circ} 9^{\circ}\right)$, and detected lower signal at STEREO-A. Our results on radio source locations are consistent with previous studies of the same event (Reiner et al., 2009, Martínez-Oliveros et al., 2012).

\subsection{May 2009 Type III Burst}

This Type III radio burst occurred on 2 May 2009 at around 19:30 UT. STEREOA was at $47.8^{\circ}$ west from a Sun-Earth line at 0.95 AU from the Sun while STEREO-B was located $46.9^{\circ}$ east at 1.02 AU from the Sun. Both STEREO detected a simple and isolated Type III radio burst with a starting time of about 19:30 UT (Figures 6 and 7). The same onset times suggest that radio sources are located roughly between the two STEREO. A solar flare triggering this emission has been located on the far side of the Sun from a view of the Earth. Hence we cannot retrieve its intensity and location as spacecraft embarking X-ray imagers orbit the Earth.

We have selected data points that correspond to peak fluxes for each frequency separately (Figure 8). The flux densities $S_{\mathrm{A}}$ and $S_{\mathrm{B}}$ are comparable at both spacecraft while their maxima occur at $225 \mathrm{kHz}$ and $1525 \mathrm{kHz}$ at STEREO-A and STEREO-B, respectively. The apparent source sizes $\gamma_{\mathrm{A}}$ and $\gamma_{\mathrm{B}}$ are very extended with maximum of $60^{\circ}$ at the lowest frequency channel. The wave vector directions $\theta_{\mathrm{A}} / \theta_{\mathrm{B}}$ and $\phi_{\mathrm{A}} / \phi_{\mathrm{B}}$ point roughly towards the Sun for high frequencies whereas the low frequency part of $\phi_{\mathrm{A}}$ and $\phi_{\mathrm{B}}$ shows the westward and eastward directions, respectively. The last panel of Figure 8 show the $2 \mathrm{D}$ degree of polarization which is about 0.1 which is negligible.

Figure 9 shows results of the radio triangulation between $125 \mathrm{kHz}$ and $1975 \mathrm{kHz}$ using wave vector directions during peak fluxes in the $X Y_{\mathrm{HEEQ}}$ and $X Z_{\text {HEEQ }}$ planes. Generally, source regions of higher frequencies are closer to the Sun. Results of the triangulation suggest that an electron beam triggering the Type III burst propagate along Parker spiral field lines on the far side of the Sun. We demonstrated a capability of STEREO/Waves to track radio sources located up to $1.5 \mathrm{AU}$ from the spacecraft.

\subsection{Statistical Results on GP Properties}

We have performed a statistical analysis of 152 events when one or two STEREO spacecraft observed simple and isolated Type III radio bursts. We have investigated median values of the flux density, wave vector directions, and polarization 
state. We have selected these data products during peak fluxes case by case for each frequency channel separately. The topmost panel of Figure 10 shows median values of the flux density $S$ vs. frequency. Both spacecraft observed a broad maximum at $1 \mathrm{MHz}$ being consistent with previous observations using spin-stabilized spacecraft (Weber, 1978; Dulk, Steinberg, and Hoang, 1984). This frequency corresponds to a region $\left(\approx 10 R_{\odot}\right)$, where the corona boundary is roughly located and the radial plasma density gradient changes according to the electron density model (Sittler and Guhathakurta, 1999). However, the observed maximum is shifted in the two events presented in Sections 3.1 and 3.2 with a factor of two and four, respectively. More discussion on the $1 \mathrm{MHz}$ maximum can be found in the linked paper (Krupar et al. 2014 ).

The second panel of Figure 10 displays median values of the apparent source size $\gamma$ vs. frequency. We have used the GP inversion assuming unpolarized emissions and the Gaussian radial source shape (Krupar et al. 2012). The apparent source size is very extended $\left(\gamma \approx 60^{\circ}\right)$ for low frequencies whereas it is roughly constant $\left(\gamma \approx 20^{\circ}\right)$ between $1 \mathrm{MHz}$ and $2 \mathrm{MHz}$ at both spacecraft. The highest frequency channel $(1975 \mathrm{kHz})$ is affected by the HFR switching between two modes (Section 3.1) and therefore it should not be taken into account. Our results for low frequencies are comparable with Steinberg, Hoang, and Dulk (1985), who analyzed 162 Type III radio bursts observed by the International Sun/Earth Explorer 3 (ISEE-3) and concluded that statistically $\gamma \approx 50^{\circ}$ for $f=100 \mathrm{kHz}$. However, they reported that $\gamma \approx 5^{\circ}$ for $1 \mathrm{MHz}$, which is about four-times smaller than we observe by STEREO/Waves. This discrepancy can be explained by different methods for estimating the source size. In the case of the spin-stabilized ISEE-3 spacecraft, the source size is deduced from the depth of modulation of the spinning dipole (Manning and Fainberg, 1980, Hoang et al. 1981). For STEREO/Waves, we apply SVD on auto- and cross-spectra measured by three non-orthogonal antennas. The SVD analysis above $1 \mathrm{MHz}$ is perhaps distorted by background signals resulting in increased source sizes. However, it was demonstrated for lower frequencies that the source size calculated by SVD on STEREO is about the same as on the Wind spacecraft (GP capabilities up to $1040 \mathrm{kHz}$ ) during short separation distances using the method dedicated for spinning spacecraft (Krupar et al., 2012).

Statistical results on absolute values of the differences between wave vectors and Sun - spacecraft lines out of $(|\theta|)$ and in $(|\phi|)$ the ecliptic are shown in the third and fourth panels of Figure 10, respectively. Generally, statistics on absolute value represent deviations of a given value from mean values (the Sun - spacecraft line in this case). These deviations for the lowest frequencies are significantly larger in the ecliptic plane than in the directions perpendicular to it. They occur to be twice larger for STEREO-A $\left(|\theta|_{\mathrm{A}} \approx 30^{\circ}\right.$ vs. $\left.|\phi|_{\mathrm{A}} \approx 60^{\circ}\right)$, whereas this difference for STEREO-B is about a factor of four $\left(|\theta|_{\mathrm{B}} \approx 40^{\circ}\right.$ vs. $\left.|\phi|_{\mathrm{B}} \approx 10^{\circ}\right)$. We do not have any explanation for this discrepancy yet. It might be caused by slightly different effective antenna parameters between the two spacecraft. We note that used effective directions have been retrieved by the inflight calibration for STEREO-B only and applied to STEREO-A too. However, distributions of $|\theta|$ and $|\phi|$ suggest that electrons triggering Type III bursts statistically propagate preferentially in the ecliptic (Dulk et al., 1986). 
The last panel of Figure 10 is the median value of the $2 \mathrm{D}$ degree of polarization in the polarization plane $C$ which is very low $(\approx 0.1)$. It is in an agreement with previous observations at kilometric wavelengths when Type III radio bursts have very low degree of polarization when compared to ground-based measurements (Dulk and Suzuki, 1980). A slight increase of $C$ at low frequencies $(<200 \mathrm{kHz})$ is probably induced by a larger error of the wave vector direction calculation due to extremely large apparent source sizes as the source position and its polarization are determined simultaneously (Krupar et al., 2012 ). The low values of $C$ are probably linked to the receiver noise. Therefore we conclude that observed Type III radio bursts are nearly unpolarized.

We have used the electron density model of Sittler and Guhathakurta (1999) to convert frequency channels into radial distances from the Sun assuming the fundamental emission (Figure 11). We fitted a linear model for data points below $1 \mathrm{MHz}$ (i.e. the statistical maximum flux density) by minimizing the $\chi^{2}$ error statistic. Obtained parameters of the model suggest that radio sources expand linearly as exciter electrons propagate outward from the Sun. Steinberg, Hoang, and Dulk (1985) showed that $\gamma$ increases with decreasing frequency just enough to fill a cone with apex located at the Sun with opening angle of about $80^{\circ}$ (full width to 1 /e brightness distribution). We observe the larger opening angle $\left(140^{\circ}\right)$ which can be explained by different methods used for source size calculation.

\subsection{Statistical Results on Radio Source Locations}

In order to take advantage of two point measurements we have statistically analyzed Type III radio bursts that have been observed by two spacecraft simultaneously (98 events from the total of 152 discussed in Section 3.3). The topmost panel of Figure 12 shows median values of the distance of triangulated radio sources from the Sun vs. frequency. We have used the electron density model of Sittler and Guhathakurta (1999) to assign particular frequencies to radial distances from the Sun with assumption of the fundamental (F, a red line) and harmonic ( $\mathrm{H}$, a blue line) emission. The highest frequency channel $(1975 \mathrm{kHz})$ should not be considered due to HFR switching.

The second panel of Figure 12 displays absolute differences between the data and both fundamental and harmonic models $(\Delta r)$. This deviation is largest $\left(\Delta r_{\mathrm{F}} \approx 0.8 \mathrm{AU}\right.$ and $\left.\Delta r_{\mathrm{H}} \approx 0.6 \mathrm{AU}\right)$ for the lowest frequency channels. For frequencies above $\approx 500 \mathrm{kHz}$ it is roughly constant for both models $\left(\Delta r_{\mathrm{F}}\right.$ and $\left.\Delta r_{\mathrm{H}} \approx 0.2 \mathrm{AU}\right)$. If we compare the source size with a radial diameter of $0.2 \mathrm{AU}$, it corresponds to $\gamma \approx 6^{\circ}$ when observed from a distance of 1 AU.

We have compared ratios between the data and model (the third panel of Figure 12). Median values of these ratios are denoted as dotted lines. Statistically, triangulated radio sources are respectively located five-times and three-times further from the Sun when compared to the model for the fundamental and harmonic components. Steinberg et al. (1984) have also observed radio sources at radial distances from about two-times to five-times further from the Sun using one spacecraft measurements with assumptions on the IP density profile.

Our results confirm that radio sources lie at considerably larger distances than regions with electron densities corresponding to particular frequencies (Steinberg et al. , 1984). This suggests, together with extended apparent source sizes 
(Figure 10), that Type III radio bursts suffer refraction at density gradients and/or scattering by inhomogeneities. In other words, these propagation effects blur actual locations, polarization properties, and beaming patterns of the radio sources at kilometric wavelengths (Steinberg, Hoang, and Dulk, 1985, Thejappa, MacDowall, and Kaiser, 2007).

In order to investigate magnetic field topology we calculated latitudes of triangulated radio sources in the HEEQ coordinate system:

$$
\theta_{\mathrm{HEEQ}}=\arctan \frac{Z_{\mathrm{HEEQ}}}{\sqrt{X_{\mathrm{HEEQ}}^{2}+Y_{\mathrm{HEEQ}}^{2}}} .
$$

The third panel of Figure 12 displays median values of absolute deviations from the solar equatorial plane $\left(\Delta \theta_{\text {HEEQ }}\right) v s$. frequency. Our results suggest that radio sources are statistically confined in this plane within $10^{\circ}$ in a given frequency range. Using one spacecraft measurements with assumptions on the IP density profile Dulk et al. (1986) demonstrated that radio sources often start at rather high latitudes, but then they tend to turn over towards the ecliptic plane at the lower frequencies. However, we do not observe this effect in the STEREO data. It might be related to larger uncertainties of radio source locations in the $Z_{\mathrm{HEEQ}}$ direction.

The fourth panel of Figure 12 shows uncertainties of the triangulation in the $X_{\mathrm{HEEQ}}, Y_{\mathrm{HEEQ}}$, and $Z_{\mathrm{HEEQ}}$ directions calculated as $\left|\mathbf{r}_{\mathrm{A}}-\mathbf{r}_{\mathrm{B}}\right|$ from Equation (15). The obtained values suggest that the largest uncertainties occur in the $Z_{\text {HEEQ }}$ being roughly $0.1 \mathrm{AU}$ for all frequencies. This can be expected by small separation distances between the spacecraft in this direction. We also observe increased uncertainties for the highest frequency channel $(1975 \mathrm{kHz})$ in all directions which correspond to the instrumental effects discussed in Section 3.1.

\section{Conclusions}

In this paper we present results of an analysis of solar radio emissions observed by STEREO with a focus on the GP properties. We have shown two examples of observations of Type III radio bursts from January 2008 and May 2009. The first event has been already analyzed by Reiner et al. (2009) and Martínez-Oliveros et al. (2012). We have achieved a good agreement with abovementioned studies on radio source locations. The second event occurred in May 2009 when the separation angle between the two STEREO was $\approx 90^{\circ}$ allowing to accurately triangulate radio sources. Although calculated apparent source sizes are very extended, our results indicate that electrons responsible for Type III radio bursts propagate along the Parker spiral field lines. We have also demonstrated that STEREO can be used for stereoscopic investigations of radio sources even if they are located on the far side of the Sun from the Earth's perspective.

We have performed a statistical survey of 152 isolated Type III radio bursts observed by STEREO between May 2007 and February 2013. The maximum flux density $S$ occurs at $\approx 1 \mathrm{MHz}$ (the first panel of Figure 10 and it is discussed in more details in the linked paper (Krupar et al., 2014). Statistical results on 


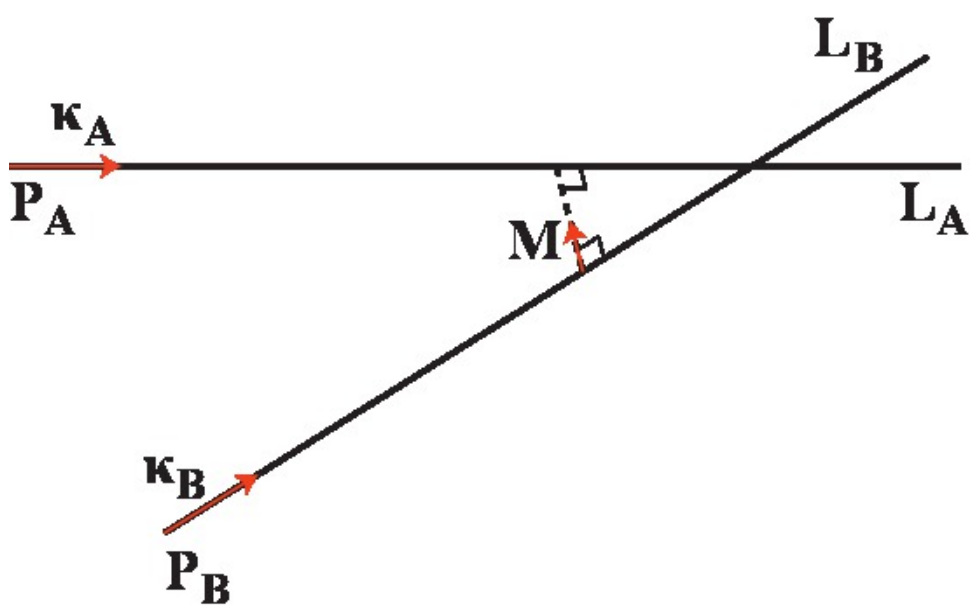

Figure 1. Illustration of a triangulation of radio sources. $\mathbf{P}_{\mathrm{A}}$ and $\mathbf{P}_{\mathrm{B}}$ are spacecraft positions. $\kappa_{\mathrm{A}}$ and $\boldsymbol{\kappa}_{\mathrm{B}}$ represent wave vector directions. These parameters form two straight lines: $\mathbf{L}_{\mathrm{A}}$ and $\mathbf{L}_{\mathrm{B}}$. The $\mathbf{M}$ is a cross product of $\boldsymbol{\kappa}_{\mathrm{A}}$ and $\boldsymbol{\kappa}_{\mathrm{B}}$.

apparent source sizes $\gamma$ which are very extended for low frequencies $\left(\gamma \approx 60^{\circ}\right)$ suggest effects of scattering by density fluctuations in the solar wind. Absolute values of deviations of wave vectors from the Sun - spacecraft line confirm that electrons responsible for Type III radio bursts statistically propagate in the ecliptic plane as it has been already observed by Dulk et al. (1986). The 2D degree of polarization is very low. Triangulated radio sources are statistically located further from the Sun with a factor of 5 and 3 for the fundamental and harmonic components, respectively. This inconsistency has been observed by Steinberg et al. (1984). It confirms that we observe only scatter images of real sources.

\section{Acknowledgements}

The authors would like to thank the many individuals and institutions who contributed to making STEREO/Waves possible. O. Kruparova thanks the support of the Czech Grant Agency grant GP13-37174P. O. Santolik acknowledges the support of the Czech Grant Agency grant GAP205/10/2279. V. Krupar thanks the support of the Czech Grant Agency grant GAP209/12/2394.

\section{References}

Bale, S.D., Ullrich, R., Goetz, K., Alster, N., Cecconi, B., Dekkali, M., Lingner, N.R., Macher, W., Manning, R.E., McCauley, J., Monson, S.J., Oswald, T.H., Pulupa, M.: 2008, The electric antennas for the STEREO/WAVES experiment. Space Sci. Rev. 136, 529. DOI ADS

Bougeret, J.-L., Fainberg, J., Stone, R.G.: 1984, Interplanetary radio storms. II - Emission levels and solar wind speed in the range 0.05-0.8 AU. Astron. Astrophys. 141, 17. ADS 


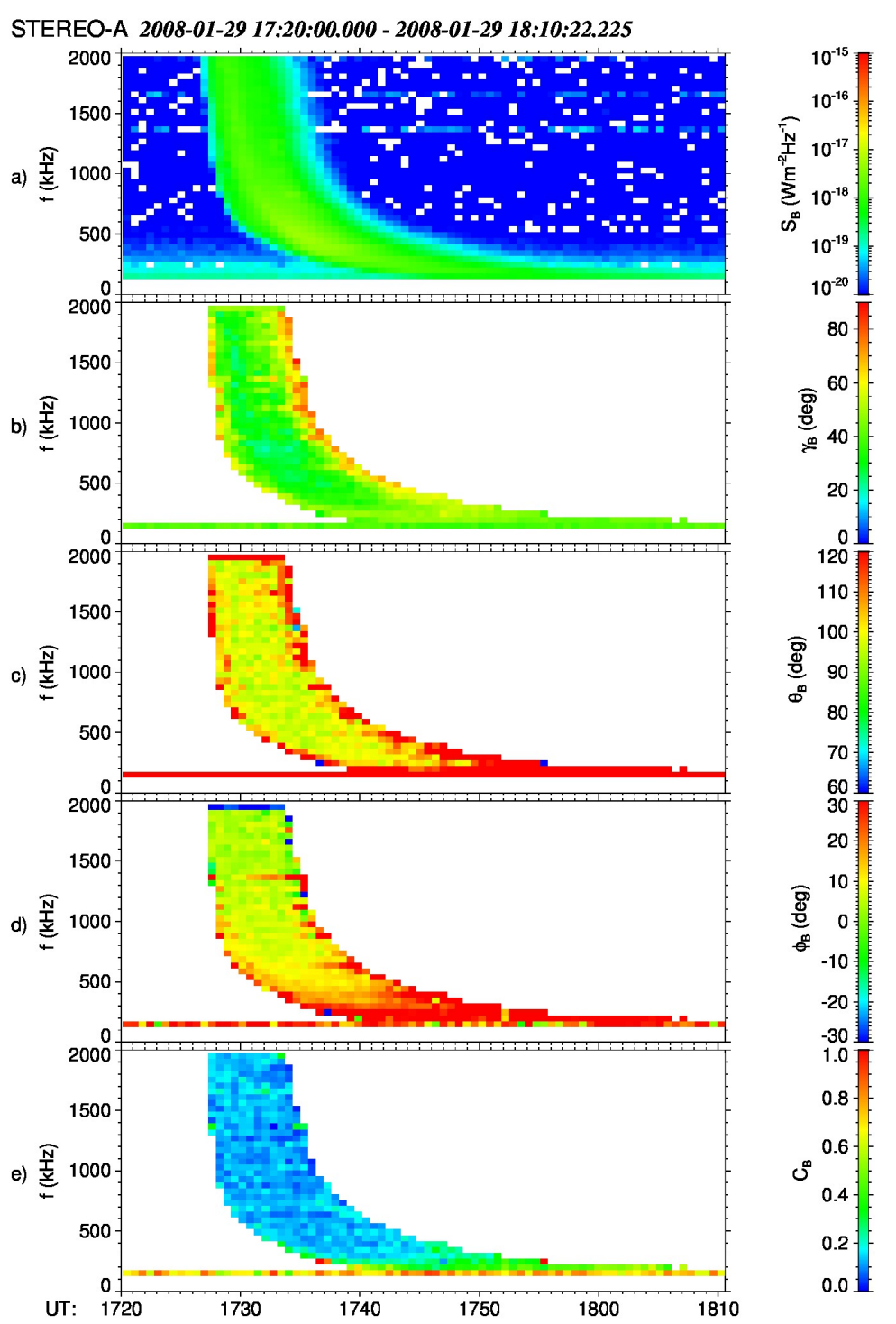

Figure 2. From 17:20 to 18:10 UT on 28 January 2008: a) flux density $S_{\mathrm{A}}$, b) the apparent source size $\gamma_{\mathrm{A}}, \mathrm{c}$ ) the colatitude $\theta_{\mathrm{A}}$ in RTN, d) the azimuth $\phi_{\mathrm{A}}$ in RTN, and e) the 2D degree of polarization $C_{\mathrm{A}}$ for STEREO-A. The intensity threshold $\left(S_{\mathrm{A}}>10^{-19} \mathrm{Wm}^{-2} \mathrm{~Hz}^{-1}\right)$ has been applied on the b), c), d) and e) panels to suppress the background.

Bougeret, J.L., Goetz, K., Kaiser, M.L., Bale, S.D., Kellogg, P.J., Maksimovic, M., Monge, N., Monson, S.J., Astier, P.L., Davy, S., Dekkali, M., Hinze, J.J., Manning, R.E., AguilarRodriguez, E., Bonnin, X., Briand, C., Cairns, I.H., Cattell, C.A., Cecconi, B., Eastwood, J., Ergun, R.E., Fainberg, J., Hoang, S., Huttunen, K.E.J., Krucker, S., Lecacheux, A., MacDowall, R.J., Macher, W., Mangeney, A., Meetre, C.A., Moussas, X., Nguyen, Q.N., Oswald, T.H., Pulupa, M., Reiner, M.J., Robinson, P.A., Rucker, H., Salem, C., Santolik, O., Silvis, J.M., Ullrich, R., Zarka, P., Zouganelis, I.: 2008, S/WAVES: The radio and plasma wave investigation on the STEREO mission. Space Sci. Rev. 136, 487. DOI ADS

Cecconi, B., Bonnin, X., Hoang, S., Maksimovic, M., Bale, S.D., Bougeret, J.-L., Goetz, K., 


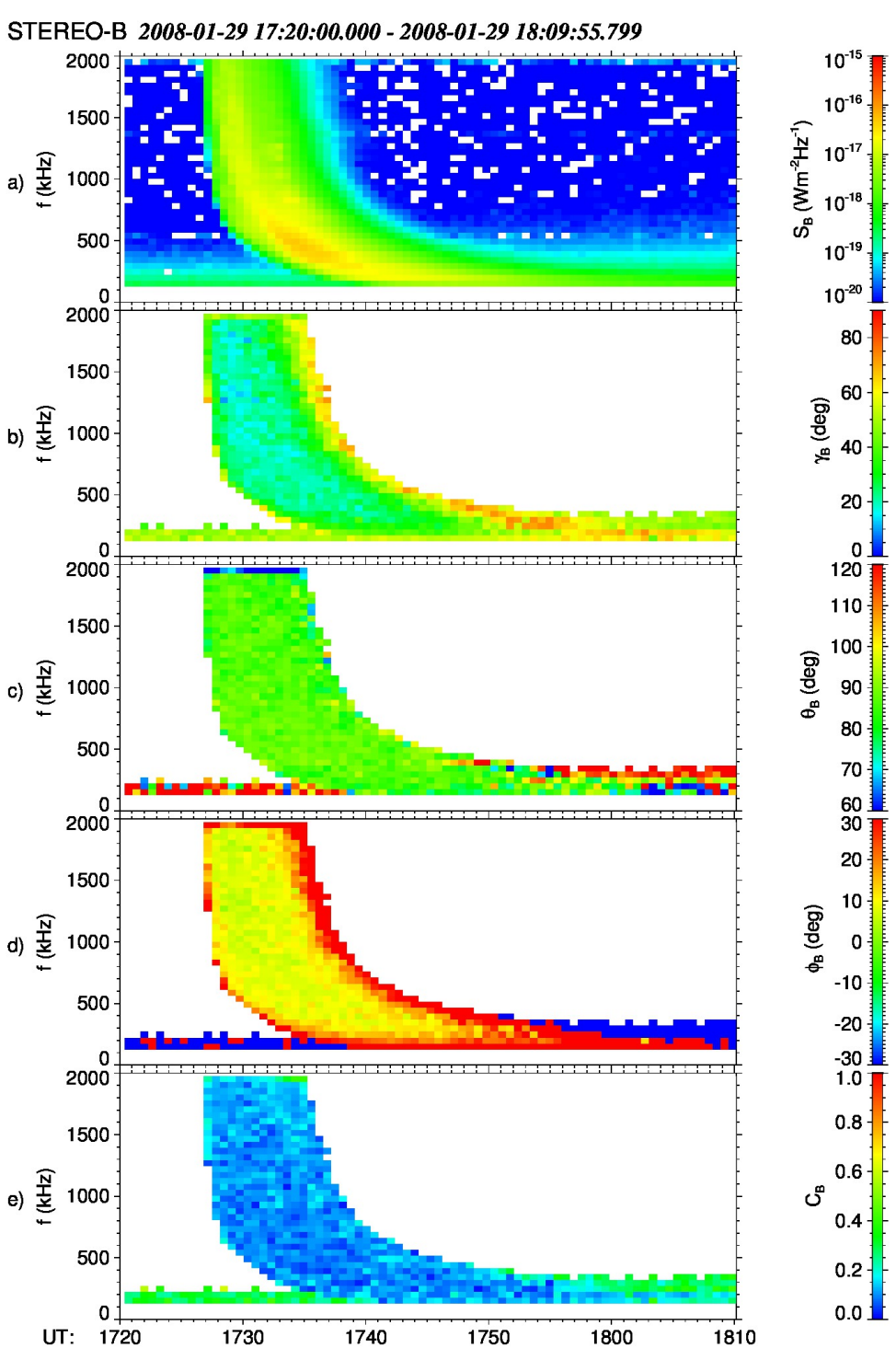

Figure 3. From 17:20 to 18:10 UT on 28 January 2008: a) flux density $S_{\mathrm{B}}$, b) the apparent source size $\gamma_{\mathrm{B}}, \mathrm{c}$ ) the colatitude $\theta_{\mathrm{B}}, \mathrm{d}$ ) the azimuth $\phi_{\mathrm{B}}$, and e) the 2D degree of polarization $C_{\mathrm{B}}$ for STEREO-B. The intensity threshold $\left(S_{\mathrm{B}}>10^{-19} \mathrm{Wm}^{-2} \mathrm{~Hz}^{-1}\right)$ has been applied on the b), c), d) and e) panels to suppress the background.

Lecacheux, A., Reiner, M.J., Rucker, H.O., Zarka, P.: 2008, STEREO/Waves goniopolarimetry. Space Sci. Rev. 136, 549. DOI ADS

Dulk, G.A., Suzuki, S.: 1980, The position and polarization of type III solar bursts. Astron. and Astrophys. 88, 203. ADS

Dulk, G.A., Steinberg, J.L., Hoang, S.: 1984, Type III bursts in interplanetary space Fundamental or harmonic? Astron. Astrophys. 141, 30. ADS

Dulk, G.A., Steinberg, J.L., Hoang, S., Lecacheux, A.: 1986, Latitude distribution of interplanetary magnetic field lines rooted in active regions. In: Marsden, R.G. (ed.) The Sun and the Heliosphere in Three Dimensions, D. Reidel Publishing Co., Dordrecht 123, 229. ADS 


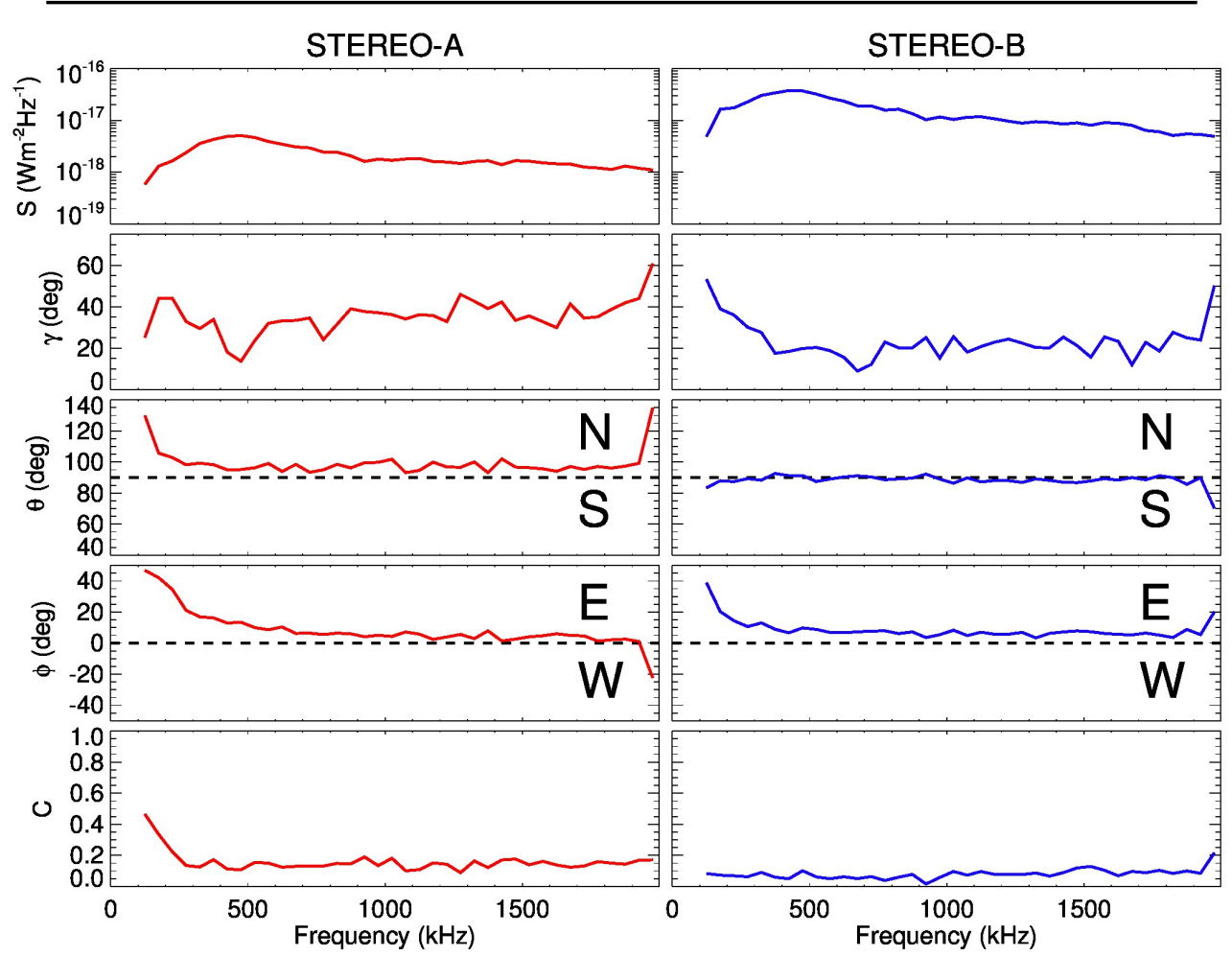

Figure 4. From 17:20 to 18:10 UT on 28 January 2008: the peak values of the flux density $S$, the colatitude $\theta$, the azimuth $\phi$, the $2 \mathrm{D}$ degree of polarization $C$, and the apparent source size $\gamma$ during peak fluxes vs frequency for STEREO-A (on the left) and STEREO-B (on the right).

Dulk, G.A., Goldman, M.V., Steinberg, J.L., Hoang, S.: 1987, The speeds of electrons that excite solar radio bursts of type III. Astron. and Astrophys. 173, 366. ADS

Fainberg, J., Evans, L.G., Stone, R.G.: 1972, Radio tracking of solar energetic particles through interplanetary space. Science 178, 743. DOI ADS

Fitzenreiter, R.J., Fainberg, J., Weber, R.R., Alvarez, H., Haddock, F.T., Potter, W.H.: 1977, Radio observations of interplanetary magnetic field structures out of the ecliptic. Solar Phys. 52, 477. DOI. ADS

Ginzburg, V.L., Zhelezniakov, V.V.: 1958, On the possible mechanisms of sporadic solar radio emission (Radiation in an isotropic plasma). Soviet Astron. 2, 653. ADS

Gurnett, D.A., Anderson, R.R.: 1976, Electron plasma oscillations associated with type III radio bursts. Science 194, 1159. DOI ADS

Hoang, S., Steinberg, J.L., Stone, R.G., Zwickl, R.H., Fainberg, J.: 1981, The 2f/p/ circumterrestrial radio radiation as seen from ISEE 3. J. Geophys. Res. 86, 4531. DOI ADS

Kaiser, M.L., Kucera, T.A., Davila, J.M., St. Cyr, O.C., Guhathakurta, M., Christian, E.: 2008, The STEREO mission: An introduction. Space Sci. Rev. 136, 5. DOI ADS.

Krupar, V., Maksimovic, M., Santolik, O., Cecconi, B., Nguyen, Q.N., Hoang, S., Goetz, K.: 2010, The apparent source size of type III radio bursts: Preliminary results by the STEREO/WAVES instruments. In: Maksimovic, M., Issautier, K., Meyer-Vernet, N., Moncuquet, M., Pantellini, F. (eds.) AIP Conf. Ser. 1216, 284. DOI ADS

Krupar, V., Santolik, O., Cecconi, B., Maksimovic, M., Bonnin, X., Panchenko, M., Zaslavsky, A.: 2012, Goniopolarimetric inversion using SVD: An application to type III radio bursts observed by STEREO. J. Geophys. Res. 117, 6101. DOI ADS.

Krupar, V., Maksimovic, M., Santolik, O., Kontar, E.P., Cecconi, B., Hoang, S., Kruparova, 


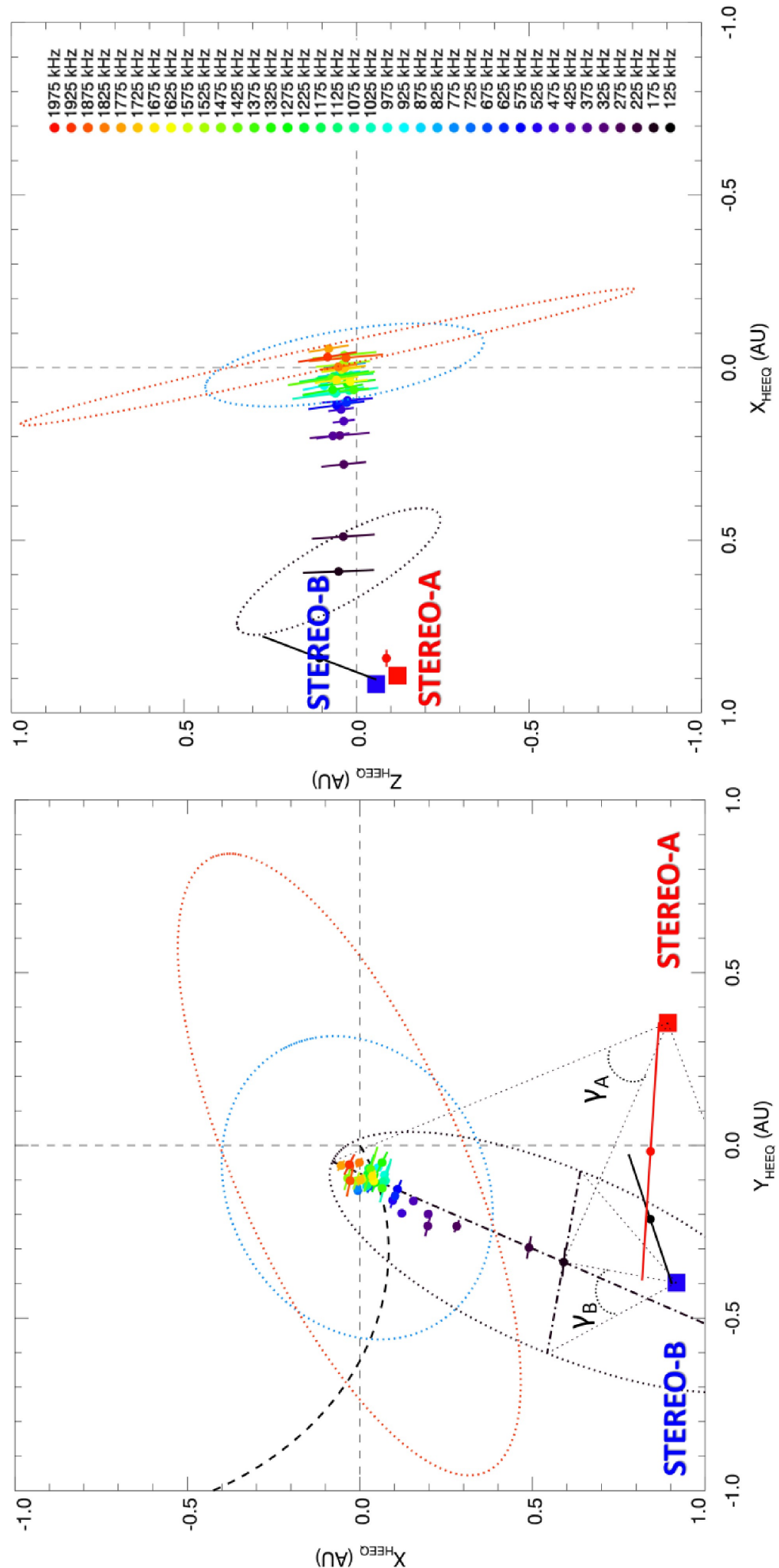

Figure 5. Propagation analysis of measurements recorded from 17:20 to $18: 10$ UT on 28 January 2008: Circles show intersections between wave vector directions (peak flux values) from STEREO-A and STEREO-B in the $X Y_{\mathrm{HEEQ}}$ and $X Z_{\mathrm{HEEQ}}$ planes. Colors denote frequency. Segment lines indicate accuracy of the triangulation. Dotted ellipses around three intersections $(175 \mathrm{kHz}, 1075 \mathrm{kHz}$, and $1925 \mathrm{kHz})$ represent apparent source sizes as seen from both spacecraft. Dot-dashed lines illustrate how the ellipse around the $175 \mathrm{kHz}$ intersection has been calculated. The black dashed line shows the Parker spiral. Positions of STEREO-A and STEREO-B are denoted by red and blue squares, respectively. The Sun is located at the center.

SOLA: krupar.tex; 6 September 2018; 2:05; p. 15 

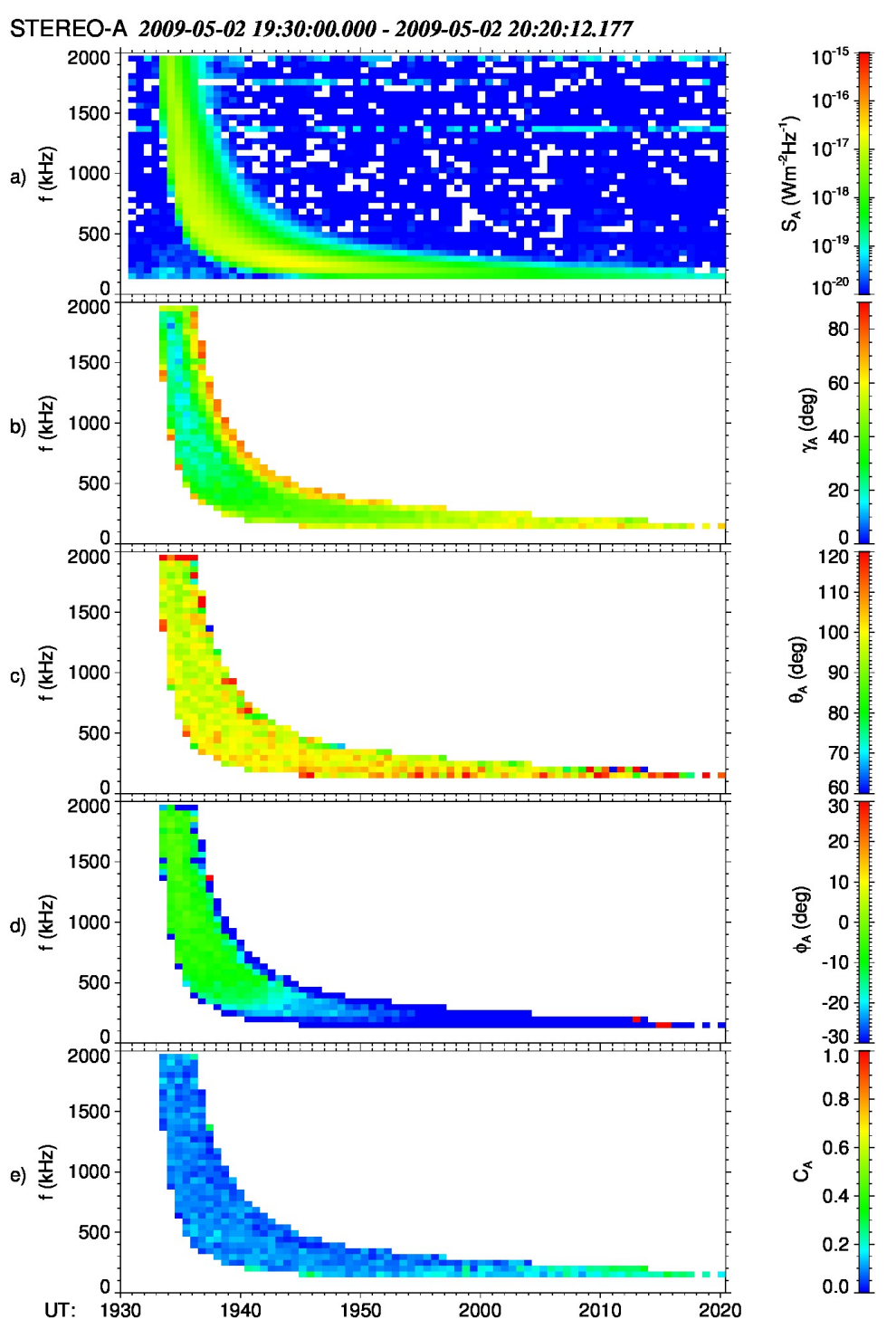

Figure 6. From 19:30 to 20:20 UT on 2 May 2009: a) the flux density $S_{\mathrm{A}}$, b) the apparent source size $\gamma_{\mathrm{A}}, \mathrm{c}$ ) the colatitude $\theta_{\mathrm{A}}, \mathrm{d}$ ) the azimuth $\phi_{\mathrm{A}}$, and e) the 2D degree of polarization $C_{\mathrm{A}}$ for STEREO-A. The intensity threshold $\left(S_{\mathrm{A}}>10^{-19} \mathrm{Wm}^{-2} \mathrm{~Hz}^{-1}\right)$ has been applied on the b), c), d) and e) panels to suppress the background.

O., Soucek, J., Reid, H., Zaslavsky, A.: 2014, Statistical survey of type III radio bursts at long wavelengths observed by the Solar TErrestrial RElations Observatory (STEREO)/ Waves instruments: Radio flux density variations with frequency. Solar Phys. 289, 3121. DOI. ADS

Lin, R.P., Potter, D.W., Gurnett, D.A., Scarf, F.L.: 1981, Energetic electrons and plasma waves associated with a solar type III radio burst. Astrophys. J. 251, 364. DOI ADS

Manning, R., Fainberg, J.: 1980, A new method of measuring radio source parameters of a partially polarized distributed source from spacecraft observations. Space Sci. Instrum. 5, 161. ADS 


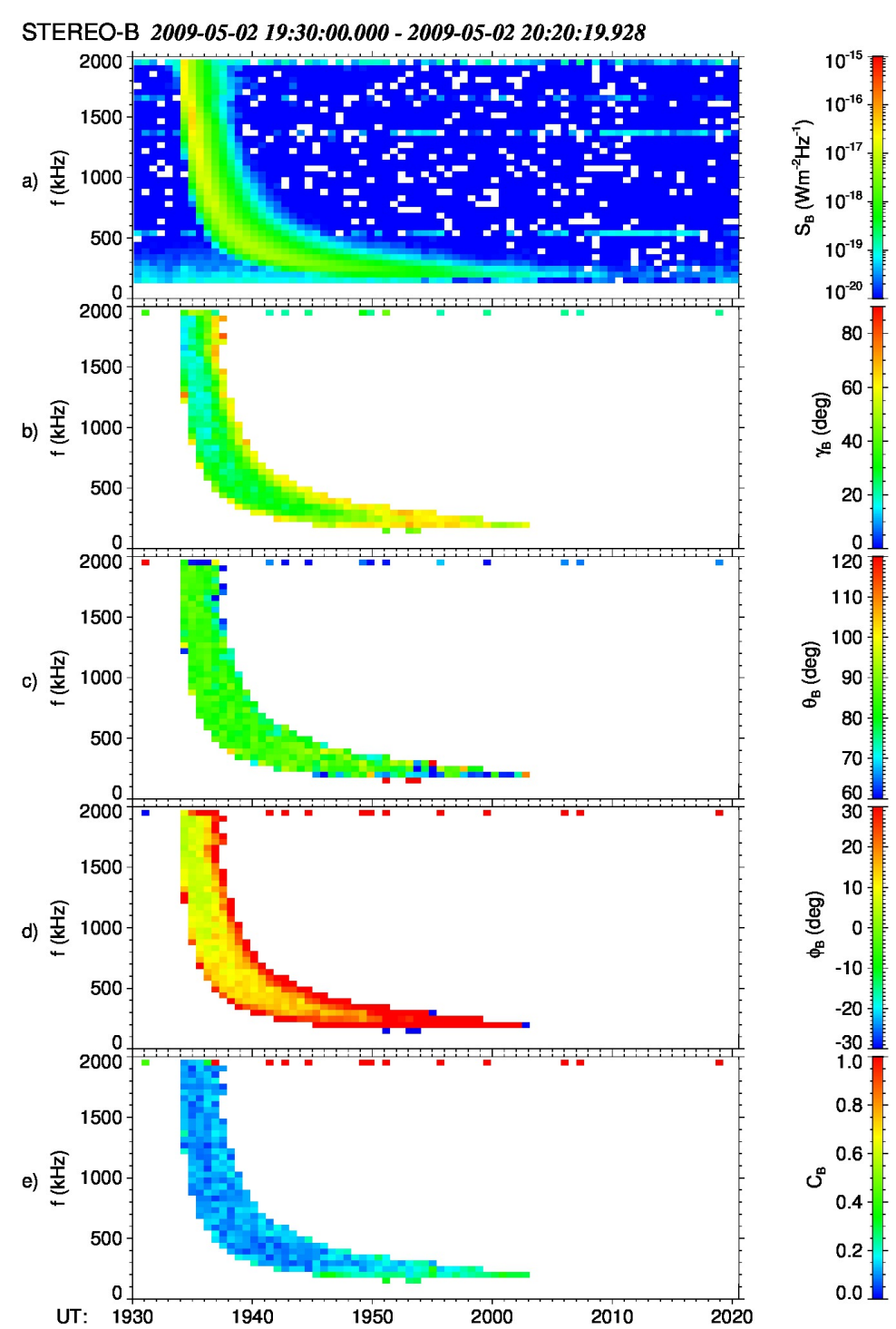

Figure 7. From 19:30 to 20:20 UT on 2 May 2009: a) flux density $S_{\mathrm{B}}$, b) the apparent source size $\gamma_{\mathrm{B}}, \mathrm{c}$ ) the colatitude $\theta_{\mathrm{B}}$, d) the azimuth angle $\phi_{\mathrm{B}}$, and e) the 2D degree of polarization $C_{\mathrm{B}}$ for STEREO-B. The intensity threshold $\left(S_{\mathrm{B}}>10^{-19} \mathrm{Wm}^{-2} \mathrm{~Hz}^{-1}\right)$ has been applied on the b), c), d) and e) panels to suppress the background.

Martens, P.C.H., Kuin, N.P.M.: 1989, A circuit model for filament eruptions and two-ribbon flares. Solar Phys. 122, 263. DOI ADS

Martínez-Oliveros, J.C., Lindsey, C., Bale, S.D., Krucker, S.: 2012, Determination of electromagnetic source direction as an eigenvalue problem. Solar Phys. 279, 153. DOI ADS

Melrose, D.B.: 1980, The emission mechanisms for solar radio bursts. Space Sci. Rev. 26, 3. DOI ADS

Parker, E.N.: 1958, Dynamics of the interplanetary gas and magnetic fields. Astrophys. J. 128, 664. DOI ADS 


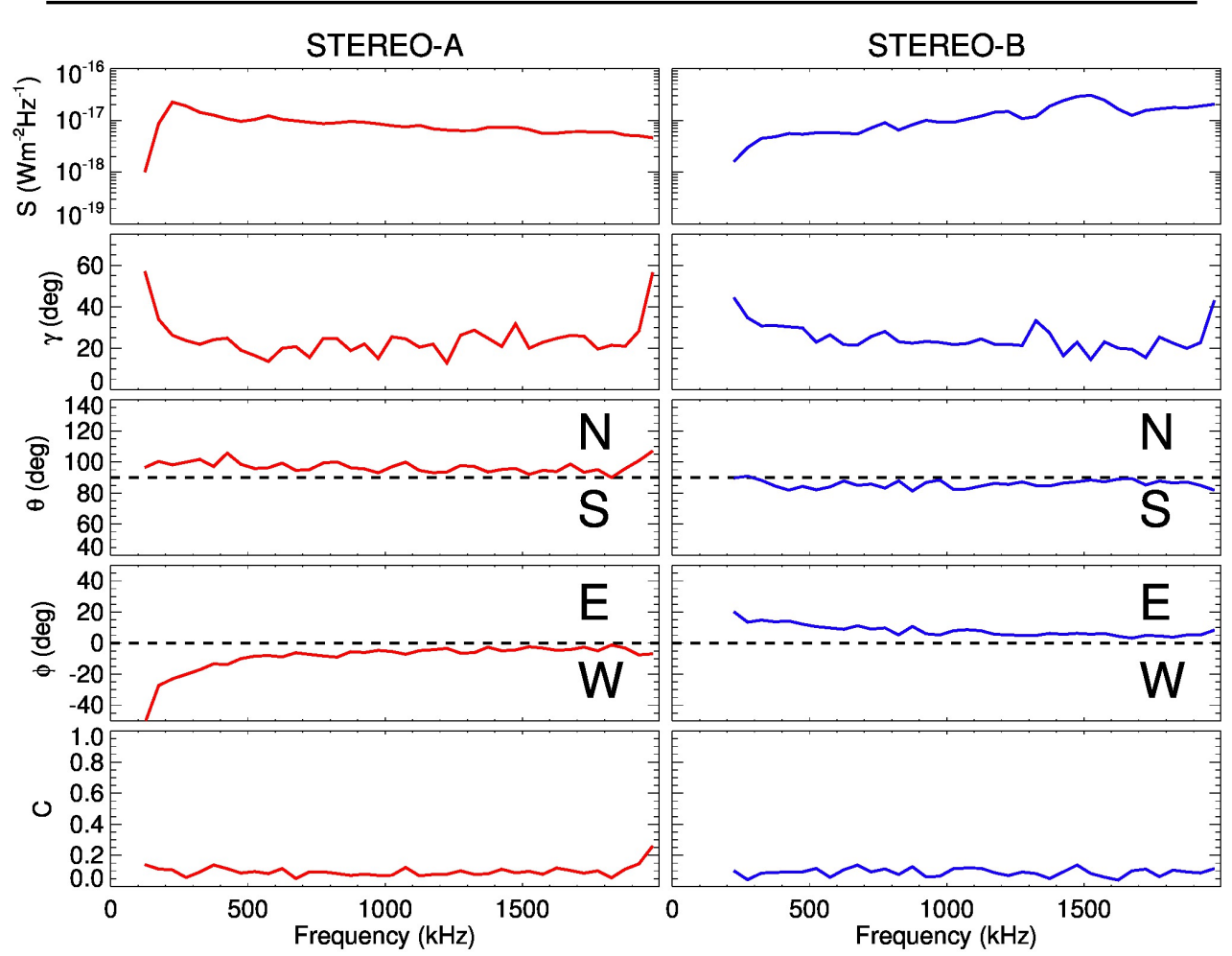

Figure 8. From 19:30 to 20:20 UT on 2 May 2009: peak values of the flux density $S$, the colatitude $\theta$ in RTN, the azimuth $\phi$ in RTN, the 2D degree of polarization $C$, and the apparent source size $\gamma$ during peak fluxes vs frequency for STEREO-A (on the left) and STEREO-B (on the right).

Reiner, M.J., Fainberg, J., Kaiser, M.L., Stone, R.G.: 1998, Type III radio source located by Ulysses/Wind triangulation. J. Geophys. Res. 103, 1923. DOI ADS

Reiner, M.J., Goetz, K., Fainberg, J., Kaiser, M.L., Maksimovic, M., Cecconi, B., Hoang, S., Bale, S.D., Bougeret, J.-L.: 2009, Multipoint observations of solar type III radio bursts from STEREO and Wind. Solar Phys., 100. DOI ADS

Santolík, O., Parrot, M., Lefeuvre, F.: 2003, Singular value decomposition methods for wave propagation analysis. Radio Sci. 38, 010000. DOI. ADS

Santolík, O., Pickett, J.S., Gurnett, D.A., Storey, L.R.O.: 2002, Magnetic component of narrowband ion cyclotron waves in the auroral zone. J. Geophys. Res. 107, 1444. DOI ADS

Sittler, E.C. Jr., Guhathakurta, M.: 1999, Semiempirical two-dimensional magnetohydrodynamic model of the solar corona and interplanetary medium. Astrophys. J. 523, 812. DOI ADS

Steinberg, J.L., Hoang, S., Dulk, G.A.: 1985, Evidence of scattering effects on the sizes of interplanetary type III radio bursts. Astron. and Astrophys. 150, 205. ADS.

Steinberg, J.L., Dulk, G.A., Hoang, S., Lecacheux, A., Aubier, M.G.: 1984, Type III radio bursts in the interplanetary medium - The role of propagation. Astron. and Astrophys. 140, 39. ADS

Thejappa, G., MacDowall, R.J., Kaiser, M.L.: 2007, Monte Carlo simulation of directivity of interplanetary radio bursts. Astrophys. J. 671, 894. DOI ADS

Weber, R.R.: 1978, Low frequency spectra of type III solar radio bursts. Solar Phys. 59, 377. DOI ADS

Weber, R.R., Fitzenreiter, R.J., Novaco, J.C., Fainberg, J.: 1977, Interplanetary baseline observations of type III solar radio bursts. Solar Phys. 54, 431. DOI ADS 

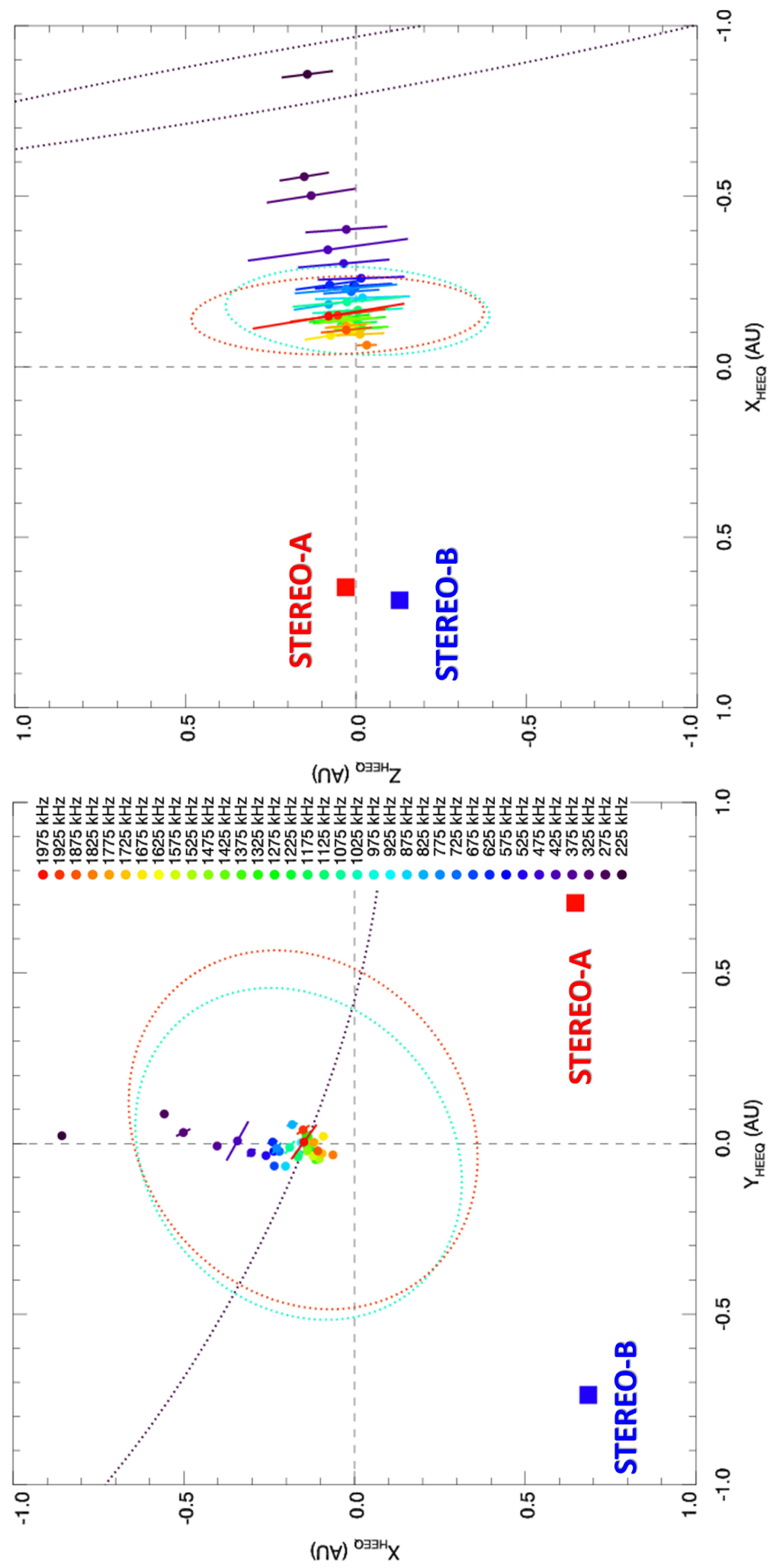

Figure 9. Propagation analysis of measurements recorded from 19:30 to 20:20 UT on 2 May 2009: Crosses show intersections between wave vector directions (peak flux values) from STEREO-A and STEREO-B in the $X Y_{\mathrm{HEEQ}}$ and $X Z_{\mathrm{HEEQ}}$ planes. Colors denote frequency. Segment lines indicate accuracy of the triangulation. Dotted ellipses around three intersections $(225 \mathrm{kHz}, 1075 \mathrm{kHz}$, and $1875 \mathrm{kHz})$ represent apparent source sizes as seen from both spacecraft. Positions of STEREO-A and STEREO-B are denoted by red and blue squares, respectively. The Sun is located at the center. 


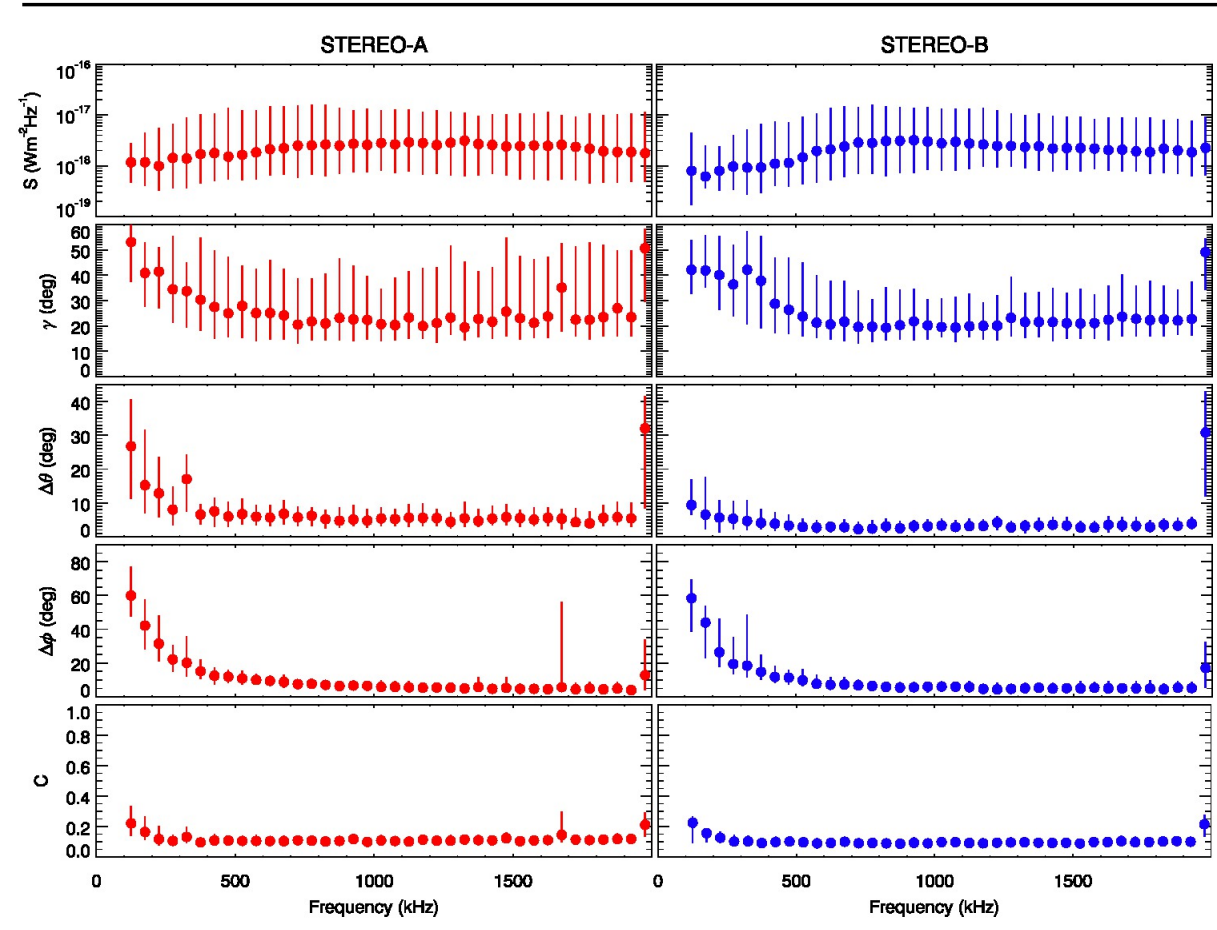

Figure 10. Results of the statistical survey of 152 Type III radio bursts obtained during peak fluxes for STEREO-A (on the left) and STEREO-B (on the right). The topmost panels display the flux density $S$. The second panels show the apparent source sizes $\gamma$. The following panels are absolute values of deviations of the wave vector from the Sun-spacecraft line out of the ecliptic (the colatitude $\theta$ ) and in the ecliptic (the azimuth $\phi$ ). The bottom panels contain 2D degree of polarization $C$. Circles are median values and errorbars represent $25 \mathrm{th} / 75$ th percentiles.

Wild, J.P.: 1950, Observations of the spectrum of high-intensity solar radiation at metre wavelengths. III. Isolated bursts. Aust. J. Sci. Res. A 3, 541. ADS

Zaslavsky, A., Meyer-Vernet, N., Hoang, S., Maksimovic, M., Bale, S.D.: 2011, On the antenna calibration of space radio instruments using the galactic background: General formulas and application to STEREO/WAVES. Radio Sci. 46, RS2008. DOI ADS 


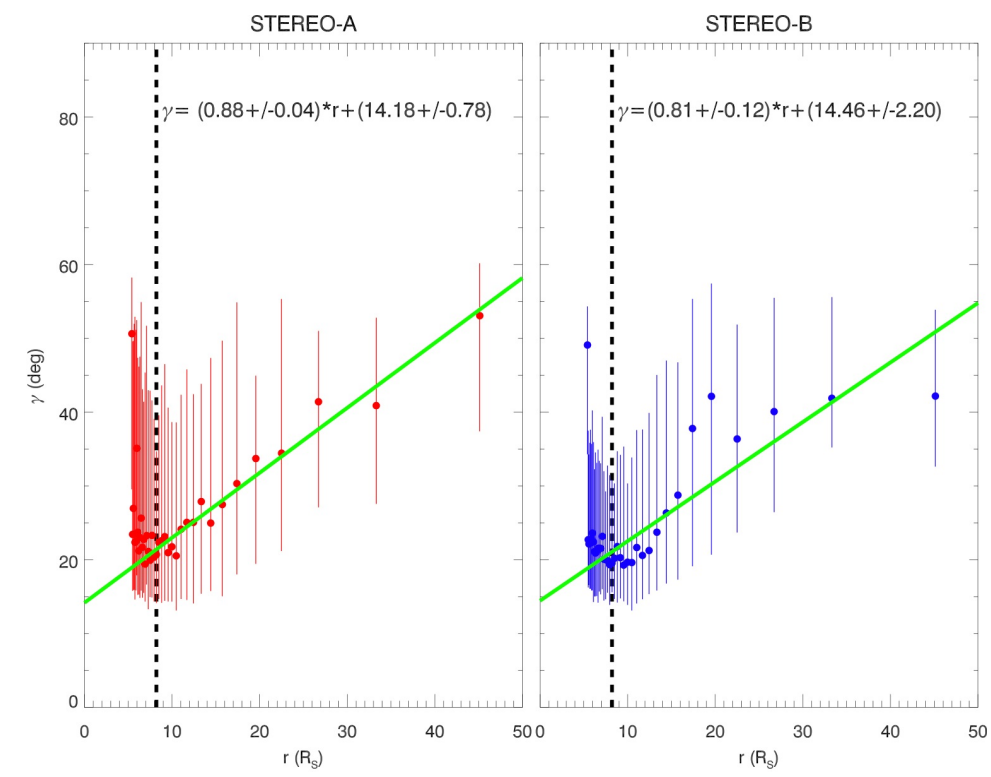

Figure 11. Results of the statistical survey of 152 Type III radio bursts obtained during peak fluxes for STEREO-A (on the left) and STEREO-B (on the right). Panels show median values of the apparent source sizes $\gamma$ as a function of a radial distance from the Sun calculated from the Sittler and Guhathakurta (1999) model with an assumption of the fundamental emission. Errorbars represent 25 th/75th percentiles. Black dashed lines indicate radial distance corresponding to $1 \mathrm{MHz}$. Green lines are linear fits of points below $1 \mathrm{MHz}$. Obtained linear model parameters with one $\sigma$ are located on the top. 

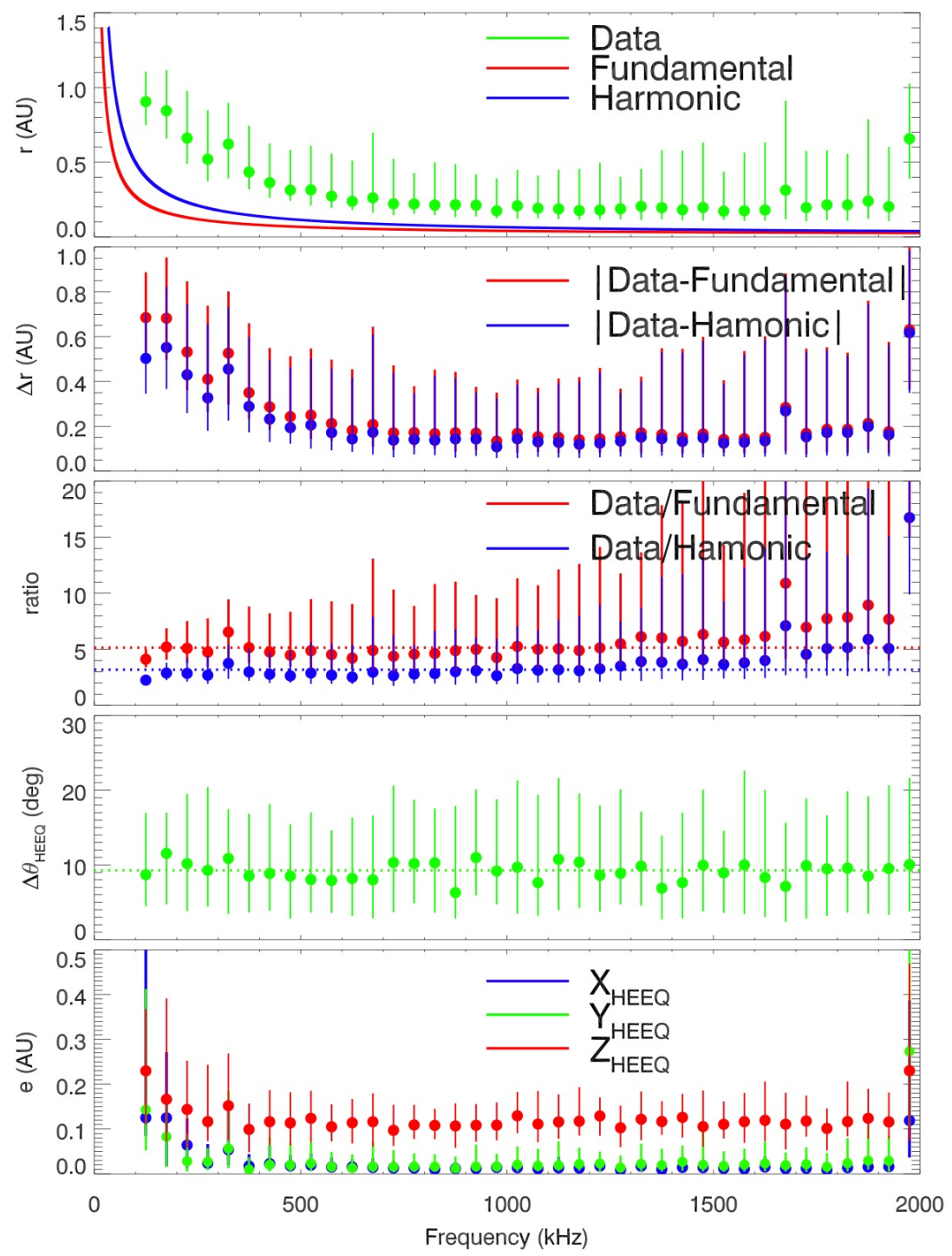

Figure 12. Results of the statistical survey of 98 Type III bursts observed simultaneously by the two STEREO. The first panel contains distances of triangulated radio sources of 98 Type III bursts vs frequency: green circles are median values and error bars represent $25 \mathrm{th} / 75 \mathrm{th}$ percentiles. The red and blue lines show the theoretical distances using the electron density model of Sittler and Guhathakurta (1999) for the fundamental and harmonic component, respectively. The second panel displays absolute differences between observed radio locations and the model. The third panel is ratio between observed radio locations and the model. Dotted lines show the median values of these ratios. The fourth panel displays median values of radio source latitudes calculated in HEEQ. The last panel contains medians of uncertainties of the triangulation $e_{\mathrm{i}}$ [Equation (15)] in the $X_{\mathrm{HEEQ}}, Y_{\mathrm{HEEQ}}$, and $Z_{\mathrm{HEEQ}}$ directions. 